\title{
Design of an electronic breastplate for automatic detection of 4 types of techniques of TaeKwonDo
}

\author{
Adrián Castañeda ${ }^{1}$, Enrique García $^{1}$, Ernesto Limón $^{1}$, and Victor Vázquez ${ }^{1}$ \\ UPIITA - IPN, Electronics Department \\ D.F., MX, 5729-6000 ext. 56882 \\ acastanedag@ipn.mx, egarciat@ipn.mx, balam_9099@hotmail.com, \\ roberto.tellez.00@gmail.com \\ http://www.upiita.ipn.mx
}

\begin{abstract}
This paper presents the design of an electronic breastplate for automatic detection of 4 types of techniques taekwondo in the thoracic section of the body (Bandal Chagui, Yop Chagui, Tuit Chagui y MontongChirugui); in order to discriminate if an impact should be considered as valid and transmit it wirelessly to a computer with a program to display the marker For the development of this work biomechanical analysis of the 4 different techniques in 10 different athletes were performed with 5 XSENS inertial sensors IMU of 9 degrees of freedom by using only the 3 axes acceleration data and finding the Pearson correlation index for each; subsequently the detection algorithm was implemented in an embedded system whit an ATM328 microcontroller in an Arduino UNO tarjet, ADXL345 accelerometer, XB24AWIT Zigbee radio and a computer program in Visual $\mathrm{C} \#$ for counting points. The device has $3.53 \times 8.53 \times 7.76 \mathrm{~cm}$ in dimensions and weighs 180 grams. Operates on a 9 volt battery that lasts 50 minutes in active mode. The communication range is up to $50 \mathrm{~m}$ and has a maximum time following the impact response of $250 \mathrm{~ms}$.
\end{abstract}

Keywords: TaeKwonDo, embedded system, IMU, computer interface

\section{Introduction}

The Taekwondo is a Korean martial art that has been developed like a sport. Its popularity is due to the method to acquire greater fitness, being a self-defense method and to become an Olympic sport. Taekwondo has a variety of techniques where their movements in a competition are made with legs and additionally with hands. Due to its scoring system where victory is obtained by knocking out the opponent or getting the mayor quantity of points, practitioners of Tae Kwon Do prefer to use techniques that allow them to easily rate and gives them a greater advantage to win the competition.

The science that studies the different sports is the sport biomechanics. Sobarzo P. defines as: Sports biomechanics is responsible for conducting studies to sports practices to search for better performance, development of technical, 
training and designing accessories for different disciplines. The overall objective of the sports biomechanics research is to develop a detailed understanding of the specific motor sports and performance variables to improve performance and reduce the incidence of injury. This results in the investigation of the specific techniques of sport, design a better sports equipment, apparel, and identify practices that predispose to injury [1].

Taekwondo practitioners typically have a large arsenal of kicks. Some biomechanical studies have focused on the techniques of upper body and others on the lower limb [2]. Peter et al [3] compared the straight punch forward from two different initial positions; the clear hand taekwondo straight punch in reverse position from the forward position was studied in relation to its standard execution model and to their success and power [4]. Hwang analyzed whip front kick to investigate patterns of muscle forces pairs of hip, knee, and ankle and the amplitudes of the segmental movements relative to the pairs of effective muscle forces applied [5]. Wohlin was interested in the kinetics and angular kinematics of the body during the kick and studied American athletes of taekwondo during the execution of the hook kick with rotation [6]. The spinning back kick was studied by Serina and Lieu focusing on the speed developed during the kick [7].

Luigi T. said that Most biomechanical investigations have tried taekwondo kicks, which can be broadly classified into three groups: linear and circular rotating. Linear kicks are the simplest in terms of mechanical efficiency analysis [2] and Falco P. concludes that Despite the high relevance of leg techniques in Taekwondo, there are, to date, few biomechanical studies to help us understand the effectiveness thereof. From a biomechanical perspective, the ability to be analyzed in relation to the strength and time, but also in relation to space [8].

Existing electronic breastplates are extremely expensive and therefore are only used in official competitions; however, have caused controversy, as discussed for the taekwondones and coaches, the benefits are not as many, because you never know when it will give a good point. As the athlete Uriel Avigdor Adriano (bronze medalist at the Pan American Games) said: Like it or not, we have to use It. It is a legal method because no longer much involved judges, rather than decisions, but sometimes accuracy is questionable, because impact whit force in the breastplate and it no mark the point and sometimes mark with a grazed [9].

The proposed device is to generate a monitoring system that is able to tell if a hit is valid, without having to use a second sensor in the Foot Guards, using sensors only in breastplate and looking for patterns in the different types of attacks, to know if this hit as it should be. The proposed design aims to reduce the cost of equipment, making it available to a larger group of people, allowing competitors to get used to using the equipment, getting adequate training to provide the athlete knowledge if the blows are impacting the right way.

\section{Meterials and Methods}

We consulted with Professor Jos Luis Onofre (coach of the national team Taekwondo 2008) and Prof. Carlos Gerardo Hernndez Segura (coach Taekwondo CE- 
CyT \# 2 Miguel Bernard Perales, interpolitécnico champion) on the techniques used to have a good development, both agreed that the most important thing is the basics, so hitting techniques thoracic are selected: Bandal Chagui, Yop Chagui, and Montong Chirugui and Tuit Chagui.

As the device detects the impact on the person receiving it is considered using accelerometers to measure the transfer of impact since this would allow the system to be relatively independent of the type and shape of the breastplate, and avoid the need to alter the current structure of the conventional breastplates and the ability to characterize the impact through the waveform obtained techniques accelerometer received and thus preventing false positive results in the system.

\subsection{Testing Protocol}

For testing protocol took into account the following control variables: Folio, date and time of the measurement, and number block replay, Name, Age, Level, Height, Weight, Gender, Type of kick, Target Distance, practicing time.

For measurement of velocity and acceleration of the kicks, X-sens MTx sensors were placed in the iliac crest, knee joint and ankle; for the fist in the shoulder joint, elbow and carpal joint. To measure the transfer of impact on the breastplate one MTi-G sensor X-sens on the left strap is placed. The accelerometers are set taking into account anatomical position in the sagittal plane watching from the right side of the test subjects. See figure 1 and 2 .

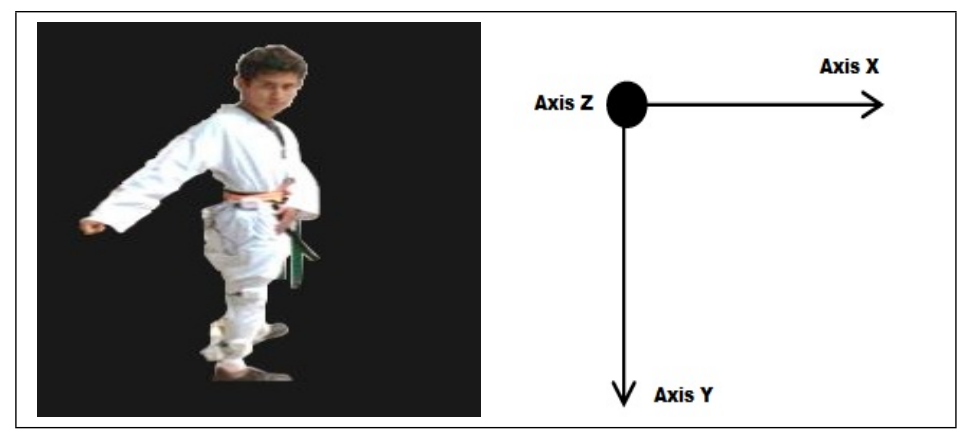

Fig. 1. Accels placed in test subject and orientation axis

It had the participation of 10 athletes, 1 yellow, 1 Advanced Green, 4 Blue, 2 Red and 2 Black 2nd Dan. To the person who made the impact was named attacker, while the one who is impacted due to technique was called receptor.

Once installed sensors on the attacker and receiver and the control variables captured the system is configured to acquire 50 samples per second for each axis of the accelerometer, the attacker subsequently stated the type of technique as the stroke, same as should be performed as quickly as possible. In each four 


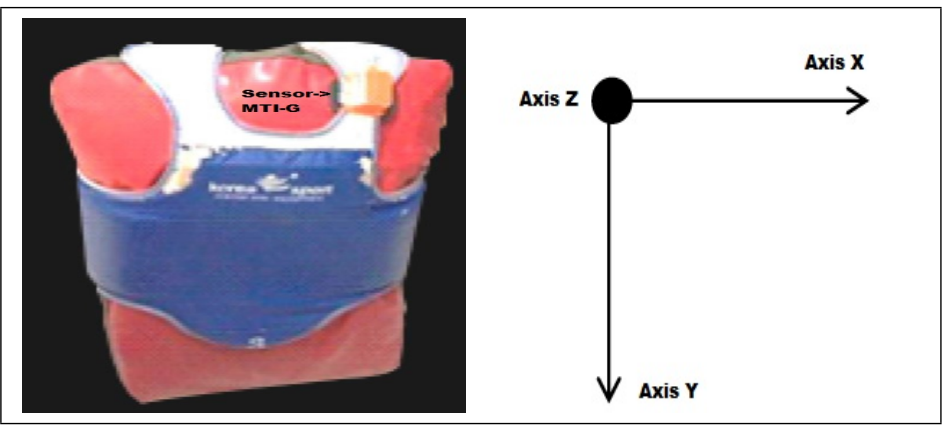

Fig. 2. Accels placed in breastplate and orientation axis

prior art attempts were made in order to test the subject to understand the procedure. As a teacher 2nd Dan finds itself it was well executed. After testing the 10 records every stroke is stored with a wait interval of 30 seconds between each, with a rest period of 5 minutes for the change of technique.

\subsection{Biomechanical Analysis}

In figure 3 the graph of acceleration in the receiver at the time of contact of a Tuit Chagui technique is shown, where you can see an abrupt change in $-64 \mathrm{~m} / \mathrm{s}^{2}$ in an interval of $2 \mathrm{~ms}$.

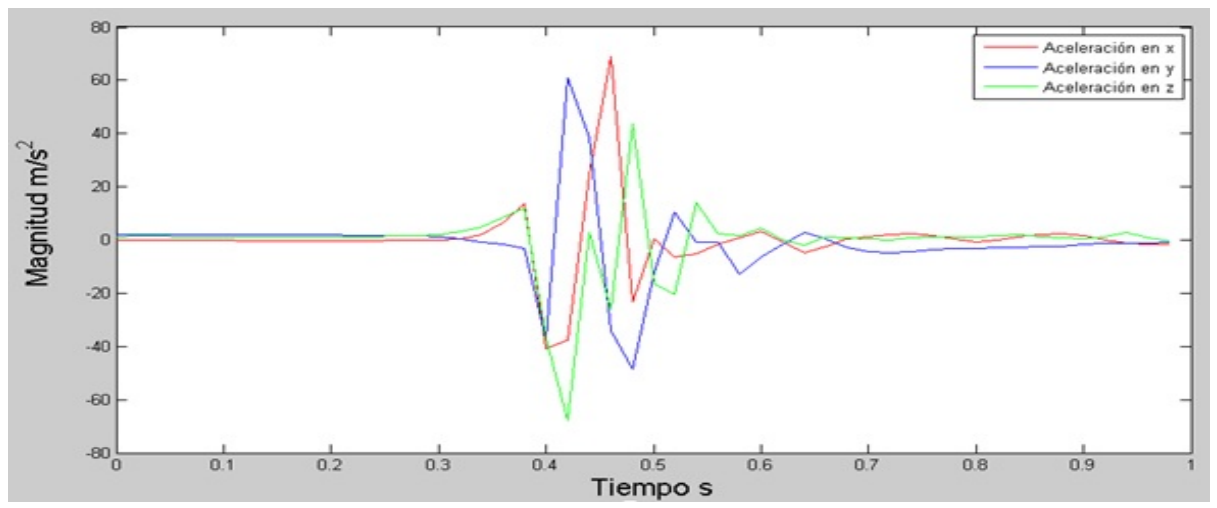

Fig. 3. Accelerometer signals in TuitChagui impact

In each of the 10 records per technic per athlete average in the receiver accelerations at impact was made, obtaining a detection threshold for the al- 
gorithm. In Bandal Chagui $-60.225 \mathrm{~m} / \mathrm{s}^{2}$, Yop Chagui $-41 \mathrm{~m} / \mathrm{s}^{2}$, Tuit Chagui $-61-67 \mathrm{~m} / \mathrm{s}^{2}$ y MontongChirugui $-54.56 \mathrm{~m} / \mathrm{s}^{2}$.

For analysis of the signals proceeded to tie all the signals according to the first peak when the punch is received and a window of $120 \mathrm{~ms}$ is taken (6 samples). See figure 4.

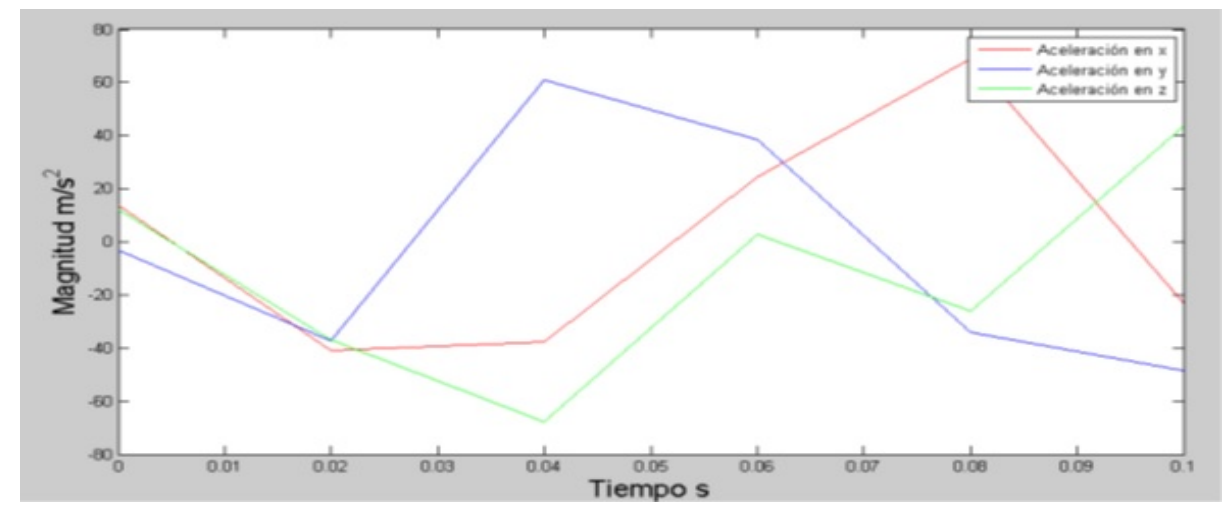

Fig. 4. Accelerometer signals in TuitChagui impact

Thereafter, proceeded to perform the correlation of each of the signals obtained to observe the degree of similarity or difference between each of them, which is calculated using the Pearson sample correlation coefficient

$$
r=\frac{\Sigma_{n}\left(A_{n}-\bar{A}\right)\left(B_{n}-\bar{B}\right)}{\sqrt{\left[\Sigma_{n}\left(A_{n}-\bar{A}\right)^{2}\right]\left[\Sigma_{n}\left(B_{n}-\bar{B}\right)^{2}\right]}}
$$

The following tables show the Pearson correlations for a person in the Tuit Chagui technique.

Table 1. Subject $1 \mathrm{X}$ axis correlation. Tuit Chagui technique

\begin{tabular}{ccccccc}
\hline $\mathbf{-}$ & $\mathbf{1}$ & $\mathbf{2}$ & $\mathbf{3}$ & $\mathbf{4}$ & $\mathbf{5}$ & $\mathbf{6}$ \\
\hline $\mathbf{1}$ & 1 & 0.844 & 0.8844 & 0.8889 & 0.6344 & 0.9525 \\
\hline $\mathbf{2}$ & 0.844 & 1 & 0.9666 & 0.9627 & 0.9049 & 0.7896 \\
\hline $\mathbf{3}$ & 0.8844 & 0.9666 & 1 & 0.9982 & 0.8355 & 0.874 \\
\hline $\mathbf{4}$ & 0.8889 & 0.9627 & 0.9982 & 1 & 0.8101 & 0.8873 \\
\hline $\mathbf{5}$ & 0.6344 & 0.9049 & 0.8355 & 0.8101 & 1 & 0.506 \\
\hline $\mathbf{6}$ & 0.9525 & 0.7896 & 0.874 & 0.8873 & 0.506 & 1 \\
\hline- & - & - & - & - & - & \\
\hline
\end{tabular}


Table 2. Subject $1 \mathrm{Y}$ axis correlation. Tuit Chagui technique

\begin{tabular}{ccccccc}
\hline $\mathbf{-}$ & $\mathbf{1}$ & $\mathbf{2}$ & $\mathbf{3}$ & $\mathbf{4}$ & $\mathbf{5}$ & $\mathbf{6}$ \\
\hline $\mathbf{1}$ & 1 & 0.9571 & 0.826 & 0.815 & 0.8988 & 0.6156 \\
\hline $\mathbf{2}$ & 0.9571 & 1 & 0.8799 & 0.8493 & 0.9336 & 0.7017 \\
\hline $\mathbf{3}$ & 0.826 & 0.8799 & 1 & 0.99 & 0.9805 & 0.4092 \\
\hline $\mathbf{4}$ & 0.815 & 0.8493 & 0.99 & 1 & 0.9578 & 0.3894 \\
\hline $\mathbf{5}$ & 0.8988 & 0.9336 & 0.9805 & 0.9578 & 1 & 0.4382 \\
\hline $\mathbf{6}$ & 0.6156 & 0.7017 & 0.4092 & 0.3894 & 0.4382 & 1 \\
\hline- & - & - & - & - & - & \\
\hline
\end{tabular}

Table 3. Subject $1 \mathrm{Z}$ axis correlation. Tuit Chagui technique

\begin{tabular}{ccccccc}
\hline $\mathbf{-}$ & $\mathbf{1}$ & $\mathbf{2}$ & $\mathbf{3}$ & $\mathbf{4}$ & $\mathbf{5}$ & $\mathbf{6}$ \\
\hline $\mathbf{1}$ & 1 & 0.509 & 0.7097 & 0.899 & -0.2028 & 0.8451 \\
\hline $\mathbf{2}$ & 0.509 & 1 & 0.5439 & 0.5429 & 0.4586 & 0.1655 \\
\hline $\mathbf{3}$ & 0.7097 & 0.5439 & 1 & 0.9332 & 0.4812 & 0.3291 \\
\hline $\mathbf{4}$ & 0.899 & 0.5429 & 0.9332 & 1 & 0.1667 & 0.6255 \\
\hline $\mathbf{5}$ & -0.2028 & 0.4586 & 0.4812 & 0.1667 & 1 & -0.6103 \\
\hline $\mathbf{6}$ & 0.8451 & 0.1655 & 0.3291 & 0.6255 & -0.6103 & 1 \\
\hline- & - & - & - & - & - & \\
\hline
\end{tabular}

Whit this correlation tables the signals that have a higher correlation than $50 \%$ in all three axesaxes were averaged to obtain a pattern signal; likewise, Pearson correlation was made for each athlete for each technique to obtain a general pattern per technique for all the subjects. Below in figure 5 the signal pattern for all individuals in the art of Tuit Chagui is shown.

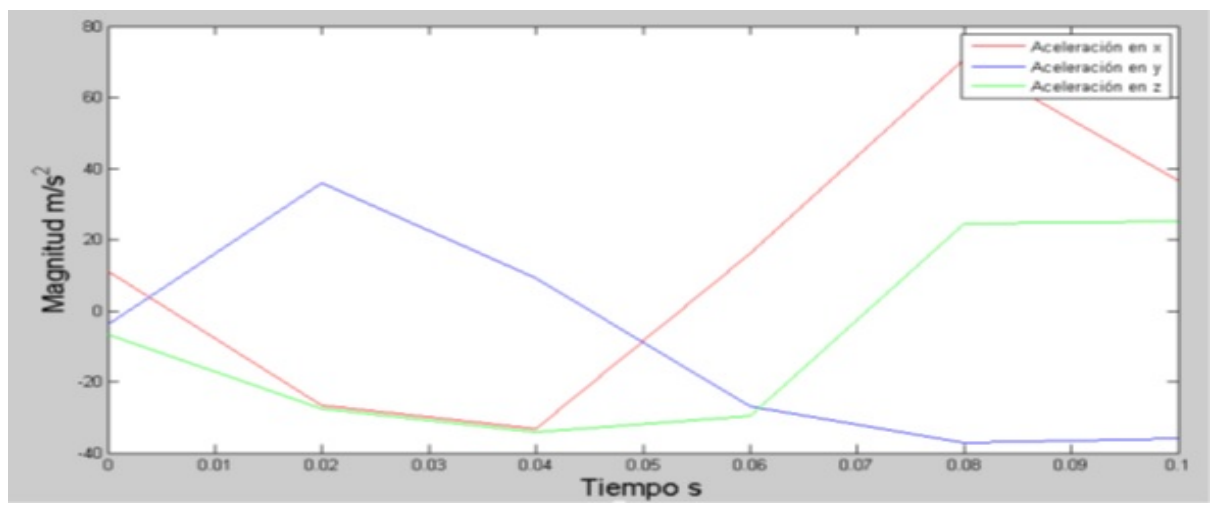

Fig. 5. Pattern signals in TuitChagui technique 


\subsection{Development of the system's hardware}

Then the figure 6 is observed where the module is displayed with the ADXL345 accelerometer and XBee connected to the microcontroller in the Arduino card and XBee works as a receiver connected to the PC.

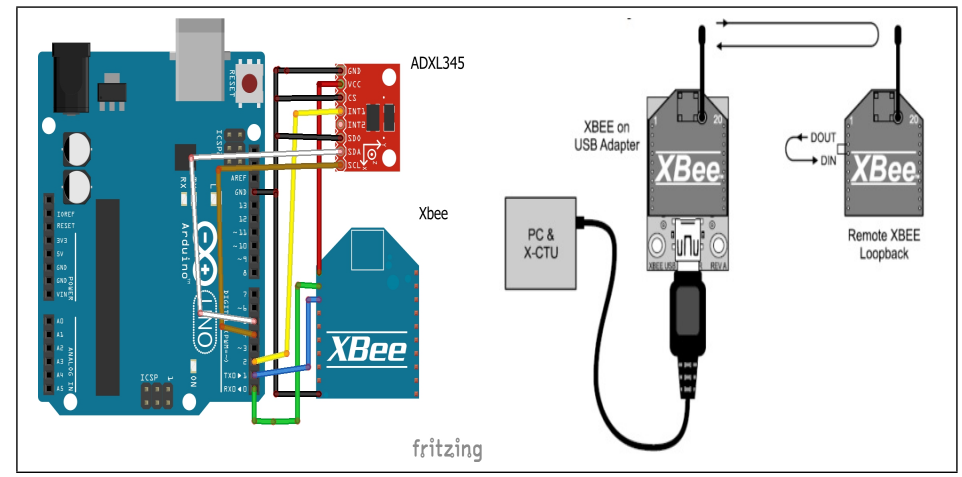

Fig. 6. Electronic circuit connections.

\subsection{Development of the system's software}

Through the Arduino platform code able to get the accelerometer data every $2 \mathrm{~ms}$ for $120 \mathrm{~ms}$ of ADXL345 sensor was programed as scheduled interruptions. Using these data proceed to perform the correlation signals with pattern (previously obtained in the biomechanical analysis) to determine if the impact point should be recognized as a point, and send a code via the zigbee module so that the data can be received and interpreted by the computer software platform developed under the Visual $\mathrm{C}$ \# to thereby a corresponding increase or no increase in the competitor's score is performed. The main program waits for the ADXL345 accelerometer tells the threshold is exceeded to begin to be considered an impact. When an impact is detected can be considered starts evaluating through the following algorithm:

1. Impact Validation

2. Pearson correlation calculation

3. Technique comparison

4. Technique validation

5. Interface comunication

The computer program figure 7 , receives a code breastplate ordering to the scoreboard to increase the score, taking the impact as valid. 


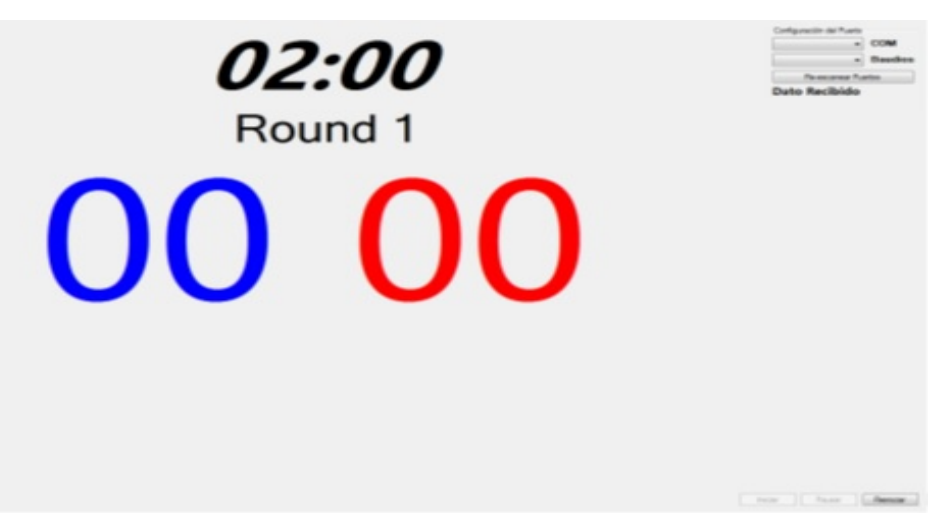

Fig. 7. PC program

\section{Results}

Was developed and built a device which measures $3.53 \mathrm{~cm}$ x $8.53 \mathrm{~cm}$ x $7.76 \mathrm{~cm}$ and weights $180 \mathrm{~g}$ and can be placed on the left of a conventional Korea Sport breastplate. Operates on a 9 volt battery that lasts 505 minutes in active mode. The communication range is up to $50 \mathrm{~m}$ and has a maximum time following the impact response of $250 \mathrm{~ms}$. See figure 8 .

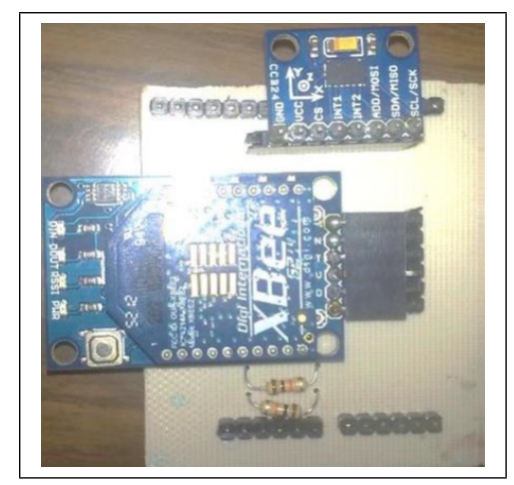

Fig. 8. Accels placed in test subject and orientation axis.

Already located in the breastplate 10 samples of each technique for a practitioner of Tae Kwon Do was captured, which were compared with the prototype samples previously proposed, getting a higher level of average correlation of $90 \%$ in all techniques. With this confirm that the data obtained with the ADXL345 sensor, are consistent with those obtained by the MTi-G sensor. 
Finally device test were made to detect how many false positives for each technique that the electronic breastplate would mark. To achieve this objetive the ten athletes launch fourteen strikes in the electronic breastplate while one 2 Dan black belt observe each technique, then in every strike we fill a table were if the technique were well executed and the PC software mark a god point we put one and if not we put zero. Subsequently an average of eache technique of the ten athletes was made, obtaining a $85 \%$ of assertiveness. Like we can see in the table 4 . One is well detected and 0 is not detected or fail.

Table 4. Subject $1 \mathrm{X}$ axis correlation. Tuit Chagui technique

\begin{tabular}{ccccc}
\hline Impact & \multicolumn{3}{c}{ Bandal Montong Tuit Yop } \\
\hline $\mathbf{1}$ & 1 & 1 & 1 & 1 \\
\hline $\mathbf{2}$ & 1 & 1 & 1 & 1 \\
\hline $\mathbf{3}$ & 0 & 0 & 1 & 1 \\
\hline $\mathbf{4}$ & 1 & 1 & 0 & 1 \\
\hline $\mathbf{5}$ & 1 & 1 & 1 & 1 \\
\hline $\mathbf{6}$ & 1 & 0 & 1 & 1 \\
\hline $\mathbf{7}$ & 1 & 1 & 1 & 0 \\
\hline $\mathbf{8}$ & 0 & 1 & 1 & 1 \\
\hline $\mathbf{9}$ & 1 & 1 & 1 & 1 \\
\hline $\mathbf{1 0}$ & 1 & 1 & 1 & 1 \\
\hline $\mathbf{1 1}$ & 1 & 1 & 0 & 1 \\
\hline $\mathbf{1 2}$ & 1 & 1 & 1 & 1 \\
\hline $\mathbf{1 3}$ & 1 & 1 & 1 & 1 \\
\hline $\mathbf{1 4}$ & 1 & 1 & 1 & 1 \\
\hline- & - & - & - & - \\
\hline Assertiveness & $85 \%$ & $85 \%$ & $85 \%$ & $92 \%$ \\
\hline & & & &
\end{tabular}

\section{Acknowledgments}

The authors thank the support from UPIITA-IPN and IPN TAEKWONDO athletes for carrying out this work.

\section{References}

1. Sobarzo, P.: Biomecánica Deportiva. El faro, 88, 8-10 (2008)

2. Luigi, T., Willy, P.: Un análisis biomecánico de la patada descendente modificada de taekwondo. Revista de Artes Marciales Asiáticas, 2, 28-39 (2007)

3. Pieter, F., Pieter, W., Heijmans, J.: Movement analysis of taekwondo techniques. Asian Journal of Physical Education, 10(3), 45-58(1987)

4. Stull, R., Barham, J.,: An analysis of movement patterns utilized by ifferent styles in the karate reverse punch in front stance. Biomechanics in Sports VI. Proceedings of the 6th International Symposium on Biomechanics in Sports, 233-243 (1990)

5. Hwang, I.: Analysis of the kicking leg in taekwondo. Biomechanics in sports III \& IV, (1987)

6. Wohlin, S.: A biomechanical description of the taekwondo turning hookkick. Unpublished Masters Thesis, Montana State University, (1989) 
Adrián Castañeda, Enrique Arturo García Tovar, Victor Roberto Vázquez Téllez et al.

7. Serina, E., Lieu, D.: Thoracic injury potential of basic competition taekwondo kicks. Journal of Biomechanics, 24(10), 951- 960(1991)

8. Falco, M.: Tesis Doctoral. Estudio sobre Parámetros Mecánicos y distancia de golpeo de Bandal Chagui en Tae Kwon Do. Universidad de Valencia, (2009)

9. Xataca México:, Tae Kwon Do y el peto electrónico en los panamericanos. http://www.xataka.com.mx/eventos-de-tecnologia/ taekwondo-y-el-peto-electronico-en-los-panamericanos, [Consulted $22 / 01 / 2014]$ 